\title{
INTÉGRALES DE RIESZ SUR UN ESPACE SYMÉTRIQUE ORDONNÉ
}

\author{
JACQUES FARAUT \\ Institut de Mathématiques de Jussieu, CNRS, UMR 7586, Université Pierre et Marie Curie \\ Case 82, 4 place Jussieu, F-75252 Paris 05, France \\ E-mail: faraut@math.jussieu.fr
}

Pour construire une solution élémentaire de l'équation des ondes Marcel Riesz utilise le prolongement analytique d'une distribution dépendant d'un paramètre complexe. Cette méthode a ensuite été étendue pour construire des solutions élémentaires d'équations plus générales. Nous allons montrer comment la méthode de Riesz peut être utilisée pour construire des solutions élémentaires d'opérateurs différentiels invariants sur un espace symétrique ordonné. Les résultats que nous exposons concernent les espaces symétriques ordonnés de Makarevic, qui ont été introduits par Bertram. La géométrie d'un tel espace est décrite à l'aide d'une algèbre de Jordan euclidienne. Le noyau de l'intégrale de Riesz vérifie une identité de type Bernstein qui est établie à l'aide de l'analyse harmonique sur l'espace symétrique riemannien dual. Ce détour ne semble pas naturel, mais ne sachant pas aujourd'hui si la transformation de Laplace sphérique est injective, nous ne connaissons pas de démonstration directe de cette identité.

Nous rappelons d'abord dans la section 1 les principales définitions relatives aux structures causales et aux espaces symétriques ordonnés. En vue de la construction des espaces symétriques ordonnés de Makarevic nous introduisons les algèbres de Jordan euclidiennes dans la section 2. Nous rappelons dans la section 3 la définition de la compactification conforme d'une algèbre de Jordan semi-simple, et celle du birapport de Kantor qui nous permettra de construire les intégrales de Riesz. Nous présentons dans la section 4 la notion d'algèbre de Jordan euclidienne involutive. Nous pouvons alors dans la section 5 présenter la construction des espaces symétriques ordonnés de Makarevic due à Bertram. L'identité de type Bernstein que vérifie le birapport de Kantor est établie dans la section

2000 Mathematics Subject Classification: 43A85, 53C35, 32M15.

Received 12 April 2001; revised 13 June 2001.

Recherche effectuée partiellement avec le soutien de la Commission Européenne (TMR 19982001 Network Harmonic Analysis).

The paper is in final form and no version of it will be published elsewhere. 
6 en utilisant la transformation de Fourier sphérique sur l'espace riemannien symétrique dual. Puis nous parvenons aux intégrales de Riesz dans la section 7. Dans la dernière section nous présentons un exemple d'espace symétrique ordonné de Makarevic : l'espace de Lobachevski imaginaire.

Ces résultats ont été présentés au séminaire Sophus Lie lors de sa réunion de Będlewo en septembre 2000 .

1. Espace symétrique ordonné. Soit $C$ un cône dans un espace vectoriel réel $V$ de dimension finie. Le cône $C$ est dit régulier s'il est convexe, fermé, pointu : $C \cap(-C)=\{0\}$, et d'intérieur non vide, ou, ce qui est équivalent, générateur : $C-C=V$. Une structure causale sur une variété $\mathcal{M}$ est la donnée d'un champ de cônes $\left\{C_{x}\right\}_{x \in \mathcal{M}}$ où $C_{x} \subset T_{x}(\mathcal{M})$. On suppose que, pour tout $x, C_{x}$ est un cône régulier et qu'il dépend continûment de $x$.

Une courbe $\gamma:[\alpha, \beta] \rightarrow \mathcal{M}$ de classe $\mathcal{C}^{1}$ par morceaux est dite causale si, pout tout $t$, la dérivée $\gamma^{\prime}(t)$ appartient au cône $C_{\gamma(t)}$ (la dérivée à droite si $\gamma^{\prime}(t)$ a une discontinuité en $t$ ). La structure causale est dite globale s'il n'existe pas de courbe causale fermée non triviale. Dans ce cas on définit une relation d'ordre sur $\mathcal{M}: x \leq y$ s'il existe une courbe causale partant de $x$ et aboutissant à $y$. L'intervalle généralisé $D(x, y)(x \leq y)$ est défini par

$$
D(x, y)=\{z \in \mathcal{M} \mid x \leq z \leq y\} .
$$

Un difféomorphisme $\varphi$ de la variété $\mathcal{M}$ est dit causal si, pour tout $x$,

$$
(D \varphi)_{x}\left(C_{x}\right)=C_{\varphi(x)} \text {. }
$$

Soit $G$ un groupe agissant sur $\mathcal{M}$ par difféomorphismes. La structure causale est dite invariante par $G$ si les transformations de $G$ sont causales. Supposons que $\mathcal{M}$ soit homogène : un groupe de Lie $G$ opère transitivement sur $\mathcal{M}$. Fixons un point de base $x_{0}$ et posons

$$
H=\left\{g \in G \mid g \cdot x_{0}=x_{0}\right\} .
$$

Alors $\mathcal{M} \simeq G / H$. Une structure causale sur $\mathcal{M}$ invariante par $G$ est déterminée par un cône $C_{x_{0}} \subset T_{x_{0}}(\mathcal{M})$ qui est invariant par $H$.

Exemples. 1. $\mathcal{M}=V$ est un espace vectoriel, $C$ est un cône régulier dans $V$, et, pour tout $x, C_{x}=C$. Les translations sont des transformations causales, et le groupe $G \simeq V$ des translations opère transitivement sur $\mathcal{M}$, et même simplement transitivement : le sous-groupe $H$ est réduit à l'élément neutre.

2. $\mathcal{M}$ est l'hyperbolö̈de d'équation

$$
\left(x^{1}\right)^{2}+\cdots+\left(x^{n}\right)^{2}-\left(x^{n+1}\right)^{2}=1 \quad(n \geq 2) .
$$

Soit $C$ le cône de $\mathbb{R}^{n+1}$ défini par

$$
\left(x^{n+1}\right)^{2}-\left(x^{1}\right)^{2}-\cdots-\left(x^{n}\right)^{2} \geq 0, x^{n+1} \geq 0,
$$

et considérons sur $\mathcal{M}$ la structure causale définie par

$$
C_{x}=T_{x}(\mathcal{M}) \cap C .
$$

Cette structure causale est globale et définit sur $\mathcal{M}$ une relation d'ordre. Les transformations de $G=S O_{0}(n, 1)$ sont causales et le groupe $G$ opère transitivement sur $\mathcal{M}$. 
Choisissons pour point de base le point $x_{0}=(1,0, \ldots, 0)$. Alors $H=S O_{0}(n-1,1)$, agissant sur les variables $x^{2}, \ldots, x^{n+1}$.

3. $\mathcal{M}$ est l'hyperbolö̈de d'équation

$$
\left(x^{1}\right)^{2}+\left(x^{2}\right)^{2}-\left(x^{3}\right)^{2}-\cdots-\left(x^{n+1}\right)^{2}=1 \quad(n \geq 2) .
$$

Le groupe $G=S O_{0}(2, n-1)$ opère transitivement sur $\mathcal{M}$. Choisissons le point de base $x_{0}=(1,0, \ldots, 0)$. Alors $H=S O_{0}(1, n-1)$, agissant sur les variables $x^{2}, \ldots, x^{n+1}$. L'espace tangent $T_{x_{0}}(\mathcal{M})$ s'identifie à

$$
\left\{x \in \mathbb{R}^{n+1} \mid x^{1}=0\right\} \simeq \mathbb{R}^{n} .
$$

Le cône $C_{x_{0}} \subset T_{x_{0}}(\mathcal{M})$, défini par

$$
\left(x^{2}\right)^{2}-\left(x^{3}\right)^{2}-\cdots-\left(x^{n+1}\right)^{2} \geq 0, x^{2} \geq 0,
$$

est invariant par $H$ et détermine sur $\mathcal{M}$ une structure causale invariante par $G$. Cette structure causale n'est pas globale. En effet le cercle défini par

$$
\gamma(t)=(\cos t, \sin t, 0, \ldots, 0) \quad(0 \leq t \leq 2 \pi),
$$

est une courbe causale fermée non triviale.

Considérons maintenant une paire symétrique $(G, H): G$ est un groupe de Lie connexe, $H$ est un sous-groupe fermé de $G$, et il existe un automorphisme involutif $\sigma$ de $G$ tel que

$$
\left(G^{\sigma}\right)_{0} \subset H \subset G^{\sigma}
$$

où

$$
G^{\sigma}=\{g \in G \mid \sigma(g)=g\},
$$

et $\left(G^{\sigma}\right)_{0}$ est sa composante neutre. L'espace symétrique associé est l'espace homogène $\mathcal{M}=G / H$. L'algèbre de Lie $\mathfrak{h}$ de $H$ est égale à

$$
\mathfrak{h}=\{X \in \mathfrak{g} \mid d \sigma(X)=X\} \quad(\mathfrak{g}=\operatorname{Lie}(G)) .
$$

L'espace tangent $T_{x_{0}}(\mathcal{M})$ au point de base $x_{0}=e H$ s'identifie à

$$
\mathfrak{q}=\{X \in \mathfrak{g} \mid d \sigma(X)=-X\} .
$$

Ainsi une structure causale invariante sur $\mathcal{M}$ est déterminée par la donnée d'un cône régulier $C$ dans $\mathfrak{q}$ qui est $\operatorname{Ad}(H)$-invariant.

Considérons un espace symétrique semi-simple ordonné $\mathcal{M}=G / H$, où $G$ est semisimple connexe de centre fini, et notons $\Gamma$ le graphe de la relation d'ordre

$$
\Gamma=\{(x, y) \in \mathcal{M} \times \mathcal{M} \mid x \geq y\} .
$$

Les intervalles $D(x, y)$ sont alors compacts. Un noyau de Volterra sur $\mathcal{M}$ est une fonction $F$ définie sur $\mathcal{M} \times \mathcal{M}$ qui est continue sur $\Gamma$ et s'annule en dehors de $\Gamma$. Le produit de deux noyaux de Volterra $F_{1}$ et $F_{2}$ est défini par

$$
F_{1} \diamond F_{2}(x, y)=\int_{\mathcal{M}} F_{1}(x, z) F_{2}(z, y) d m(z),
$$


où $d m(z)$ est une mesure sur $\mathcal{M}$ invariante par $G$. Cette intégrale est bien définie car l'intégrant s'annule en dehors de l'intervalle

$$
D(y, x)=\{z \in \mathcal{M} \mid y \leq z \leq x\}
$$

qui est compact. Un noyau $F$ est dit invariant par $G$ si $F(g \cdot x, g \cdot y)=F(x, y)$ pour tout $g \in G$. L'algèbre $\mathcal{V}(\mathcal{M})^{\#}$ des noyaux de Volterra invariants est commutative. Nous considérerons dans la section 7 la transformation de Laplace sphérique qui transforme le produit $\diamond$ de deux noyaux de Volterra invariants en produit ordinaire.

Pour ce qui concerne les espaces symétriques causaux on peut consulter [HilgertÓlafsson, 1997].

2. Cônes symétriques et algèbres de Jordan. Soit $\Omega$ un cône ouvert convexe dans un espace euclidien $V$. Le cône dual ouvert de $\Omega$ est défini par

$$
\Omega^{*}=\{x \in V \mid \forall y \in \bar{\Omega} \backslash\{0\},(x \mid y)>0\} .
$$

Le cône $\Omega$ est dit autodual si $\Omega^{*}=\Omega$. Le groupe $G(\Omega)$ des automorphismes linéaires de $\Omega$ est défini par

$$
G(\Omega)=\{g \in G L(V) \mid g(\Omega)=\Omega\} .
$$

Le cône $\Omega$ est dit homogène si le groupe $G(\Omega)$ opère transitivement sur $\Omega$. S'il est autodual et homogène, il est dit symétrique.

EXemples. 1. Le cône circulaire : $V=\mathbb{R}^{n}, \Omega$ défini par

$$
\left(x^{1}\right)^{2}+\cdots+\left(x^{n-1}\right)^{2}<\left(x^{n}\right)^{2}, x^{n}>0
$$

est symétrique.

2. Le cône des matrices symétriques (respectivement hermitiennes) définies positives, $\Omega \subset V=\operatorname{Sym}(m, \mathbb{R})$ (respectivement $\operatorname{Herm}(m, \mathbb{C})$ ), est symétrique.

Etant donné un cône symétrique $\Omega$ nous allons construire, suivant la méthode de [Bertram, 1996b, 1998], un espace symétrique causal $\mathcal{M}=G / H$ pour lequel $\mathfrak{q} \simeq V$, et $C_{x_{0}} \simeq-\bar{\Omega}$. Cette construction utilise la relation qui existe entre les cônes symétriques et les algèbres de Jordan.

Une algèbre de Jordan $V$ est un espace vectoriel muni d'un produit (c'est à dire une application bilinéaire $V \times V \rightarrow V$ ) tel que

$$
\begin{aligned}
& (J 1) \quad x \circ y=y \circ x, \\
& (J 2) \quad x^{2} \circ(x \circ y)=x \circ\left(x^{2} \circ y\right) .
\end{aligned}
$$

On écrit $x \circ y=L(x) y$, et $(J 2)$ signifie que

$$
\left[L(x), L\left(x^{2}\right)\right]=0 .
$$

Nous supposerons que $V$ est réelle et de dimension finie. L'algèbre de Jordan $V$ est dite euclidienne s'il existe sur $V$ un produit scalaire euclidien associatif :

$$
(x \circ y \mid z)=(y \mid x \circ z),
$$

c'est à dire que $L(x)$ est autoadjoint pour tout $x$. 
THÉORÈME (Koecher, Vinberg). L'intérieur $\Omega$ du cône des carrés des élements d'une algèbre de Jordan euclidienne est un cône symétrique, et tout cône symétrique est obtenu de cette façon.

Exemples. 1. Sur $V=\mathbb{R}^{n}$ on considère le produit défini par : $z=x \circ y$ si (notant les coordonnées $x^{0}, \ldots, x^{n-1}$ )

$$
\begin{aligned}
& z^{0}=x^{0} y^{0}+\cdots+x^{n-1} y^{n-1}, \\
& z^{i}=x^{0} y^{i}+x^{i} y^{0}(i=1, \ldots, n-1) .
\end{aligned}
$$

Le produit scalaire usuel sur $\mathbb{R}^{n}$ est associatif, et $V$ est une algèbre de Jordan euclidienne que nous noterons $\mathbb{R}^{1, n-1}$

2. Sur $V=\operatorname{Sym}(m, \mathbb{R})$ (respectivement $\operatorname{Herm}(m, \mathbb{C})$ ) on considère le produit défini par

$$
x \circ y=\frac{1}{2}(x y+y x) .
$$

Le produit scalaire défini par $(x \mid y)=\operatorname{tr}(x y)$ est associatif, et $V$ est une algèbre de Jordan euclidienne.

Le groupe $\operatorname{Aut}(V)$ des automorphismes de $V$ est un sous-groupe compact de $G(\Omega)$, et

$$
\operatorname{Aut}(V)=\{g \in G(\Omega) \mid g \cdot e=e\} .
$$

Supposons que l'algèbre de Jordan est simple, ou, ce qui est équivalent, que le cône $\Omega$ est irréductible. Fixons un repère de Jordan de $V$, c'est à dire un système maximal $\left\{c_{1}, \ldots, c_{r}\right\}$ d'idempotents deux à deux orthogonaux :

$$
c_{i}^{2}=c_{i}, c_{i} c_{j}=0 \text { si } i \neq j, c_{1}+\cdots+c_{r}=e .
$$

Le nombre $r$ d'idempotents d'un repère de Jordan est le rang de $V$. Tout élément $x$ de $V$ s'écrit

$$
x=k \cdot \sum_{i=1}^{r} \lambda_{i} c_{i} \quad\left(k \in \operatorname{Aut}(V), \lambda_{i} \in \mathbb{R}\right) .
$$

Les nombres réels $\lambda_{i}$ sont les valeurs propres de $x$. La trace $\operatorname{tr}(x)=\sum \lambda_{i}$ est une forme linéaire, et $\Delta(x)=\prod \lambda_{i}$ est un polynôme homogène de degré $r$ appelé déterminant. Si $\Delta(x) \neq 0, x$ est inversible et

$$
x^{-1}=k \cdot \sum_{i=1}^{r} \frac{1}{\lambda_{i}} c_{i} .
$$

L'application $j: x \mapsto-x^{-1}$ est rationnelle $(\Delta(x) j(x)$ est un polynôme à valeurs dans $V)$. Sa différentielle est donnée par

$$
(D j)_{x}=P\left(x^{-1}\right),
$$

où $P: V \rightarrow \operatorname{End}(V)$ est l'application définie par

$$
P(x)=2 L(x)^{2}-L\left(x^{2}\right),
$$

appelée représentation quadratique. Dans l'exemple 2,

$$
P(x) y=x y x .
$$


Notons que la trace, le déterminant et l'inverse d'un élément $x$ sont définis pour une algèbre de Jordan quelconque (non nécessairement euclidienne) en considérant le polynôme minimal de $x$.

Posons

$$
e_{j}=c_{1}+\cdots+c_{j}, V_{j}=\left\{x \in V \mid c_{j} x=x\right\} \quad(1 \leq j \leq r) .
$$

Le sous-espace $V_{j}$ est une sous-algèbre de $V$ de rang $j$. Le polynôme $\Delta_{j}$ défini par

$$
\Delta_{j}(x)=\operatorname{det}_{V_{j}}\left(\operatorname{pr}_{V_{j}} x\right)
$$

est appelé le $j$-ième déterminant mineur principal. Si $x \in \Omega$, alors $\Delta_{j}(x)>0$. La fonction puissance $\Delta_{\mathbf{s}}\left(\mathbf{s}=\left(s_{1}, \ldots s_{r}\right) \in \mathbb{C}^{r}\right)$ est définie par

$$
\Delta_{\mathbf{s}}=\Delta_{1}(x)^{s_{1}-s_{2}} \ldots \Delta_{r-1}(x)^{s_{r-1}-s_{r}} \Delta_{r}(x)^{s_{r}} .
$$

La fonction gamma de Gindikin d'un cône symétrique $\Omega$ est définie par

$$
\Gamma_{\Omega}(\mathbf{s})=\int_{\Omega} e^{-\operatorname{tr}(x)} \Delta_{\mathbf{s}}(x) \Delta(x)^{-\frac{n}{r}} d x .
$$

Elle s'exprime à l'aide de la fonction gamma d'Euler. Si $V$ est simple,

$$
\Gamma_{\Omega}(\mathbf{s})=(2 \pi)^{\frac{n-r}{2}} \Gamma\left(s_{1}\right) \Gamma\left(s_{2}-\frac{d}{2}\right) \cdots \Gamma\left(s_{r}-\frac{d}{2}(r-1)\right) .
$$

Le nombre $d$ est lié à la dimension $n$ et au rang $r$ par la relation

$$
n=r+\frac{d}{2} r(r-1)
$$

Concernant les résultats énoncés ci-dessus on peut consulter [Faraut-Korányi, 1994].

Nous utiliserons dans les sections 6 et 7 les formules suivantes.

Pour $\Re \alpha>-1$ et $\Re \lambda_{j}>\frac{d}{4}(r-1)$,

$$
\int_{\Omega \cap(e-\Omega)} \Delta_{\lambda+\rho}(x) \Delta(e-x)^{\alpha} \Delta(x)^{-\frac{n}{r}} d x=\frac{\Gamma_{\Omega}(\lambda+\rho) \Gamma_{\Omega}\left(\alpha+\frac{n}{r}\right)}{\Gamma_{\Omega}\left(\lambda+\rho+\alpha+\frac{n}{r}\right)} .
$$

Pour $\Re \alpha>\frac{d}{2}(r-1), \frac{d}{4}(r-1)<\Re \lambda_{j}<\alpha-\frac{d}{4}(r-1)$,

$$
\int_{\Omega} \Delta_{\lambda+\rho}(x) \Delta(e+x)^{-\alpha} \Delta(x)^{-\frac{n}{r}} d x=\frac{\Gamma_{\Omega}(\rho-\lambda+\alpha) \Gamma_{\Omega}(\rho+\lambda)}{\Gamma_{\Omega}(\alpha)} .
$$

Dans ces formules $\rho=\left(\rho_{1}, \ldots, \rho_{r}\right)$, avec

$$
\rho_{j}=\frac{d}{4}(2 j-r-1) .
$$

[Faraut-Korányi, 1994], p. 309 et 310.

3. Le birapport de Kantor. Soit $V$ une algèbre de Jordan réelle ou complexe de dimension finie. Nous supposons que $V$ est semi-simple, c'est à dire que la forme bilinéaire $\tau(x, y)=\operatorname{Tr} L(x y)$ est non dégénérée. On note $n$ sa dimension, $r$ son rang, et $\Delta$ le polynôme déterminant. Le groupe de structure de $V$, noté $\operatorname{Str}(V)$, est l'ensemble des transformations linéaires inversibles $g$ de $V$ telles que

$$
P(g \cdot x)=g P(x) g^{\prime},
$$


où $g^{\prime}$ désigne la transformation transposée relativement à $\tau$, et le polynôme déterminant est un semi-invariant de $\operatorname{Str}(V):$ pour $g \in \operatorname{Str}(V)$,

$$
\Delta(g \cdot x)=\chi(g) \Delta(x),
$$

où $\chi$ est un caractère de $\operatorname{Str}(V)$.

Le groupe conforme de $V$, noté Conf $(V)$, aussi appelé groupe de Kantor-Koecher-Tits, est le groupe des transformations rationnelles de $V$ engendré par les translations, les transformations linéaires de $\operatorname{Str}(V)$ et la transformation $j: x \mapsto-x^{-1}$. C'est un groupe de Lie dont l'algèbre de Lie $\operatorname{conf}(V)$ se compose des champs de vecteurs

$$
\xi(x)=u+T x-P(x) v,
$$

où $u, v \in V$, et $T \in \mathfrak{s t r}(V)=\operatorname{Lie}(\operatorname{Str}(V))$. Les éléments de $\operatorname{Conf}(V)$ sont appelés transformations conformes. Cette appelation est justifiée par le fait qu'en tout point $x$ où une telle transformation $g$ est définie, sa différentielle $(D g)_{x}$ appartient au groupe de structure. Ceci admet une réciproque :

ThÉORÈmE DE LiOuVILle GÉNÉRALISÉ. Supposons que $V$ ne soit isomorphe ni à $\mathbb{R}$ ni à $\mathbb{C}$. Soit $\varphi$ un difféomorphisme local de $V, \varphi: U_{1} \rightarrow U_{2}$, où $U_{1}$ et $U_{2}$ sont deux ouverts de $V$, tel qu'en tout point $x \in U_{1}$ sa différentielle $(D \varphi)_{x}$ appartienne au groupe de structure, alors $\varphi$ se prolonge en une transformation conforme.

[Bertram, 1996a], [Gindikin-Kaneyuki, 1998].

Le sous-groupe $Q=\operatorname{Str}(V) \ltimes V$ de $\operatorname{Conf}(V)$ des transformations conformes affines est un sous-groupe parabolique maximal de $\operatorname{Conf}(V)$. La variété homogène $M=\operatorname{Conf}(V) / Q$ est compacte, et est appelée compactification conforme de $V$. En effet l'application

$$
x \mapsto\left(\tau_{x} \circ j\right) Q, \quad V \rightarrow M,
$$

est un plongement d'image dense ( $\tau_{x}$ désigne la translation de vecteur $x$ ).

Le birapport de Kantor [Kantor, 1967] de quatre points de $V$ est la fonction rationnelle définie par

$$
\left\{x_{1}, x_{2}, x_{3}, x_{4}\right\}=\frac{\Delta\left(x_{1}-x_{3}\right)}{\Delta\left(x_{2}-x_{3}\right)}: \frac{\Delta\left(x_{1}-x_{4}\right)}{\Delta\left(x_{2}-x_{4}\right)} .
$$

Il est invariant par le groupe conforme agissant diagonalement : si $g \in \operatorname{Conf}(V)$,

$$
\left\{g \cdot x_{1}, g \cdot x_{2}, g \cdot x_{3}, g \cdot x_{4}\right\}=\left\{x_{1}, x_{2}, x_{3}, x_{4}\right\} .
$$

L'invariance par translation est évidente. L'invariance par $\operatorname{Str}(V)$ résulte de ce que le déterminant est un semi-invariant de $\operatorname{Str}(V)$. L'invariance par la transformation $j$ est une conséquence de la formule

$$
\Delta(j(x)-j(y))=\frac{\Delta(x-y)}{\Delta(x) \Delta(y)}
$$

([Faraut-Korányi, 1994], Lemma X.4.4). Le birapport se prolonge en une fonction de quatre points de la compactification conforme $M$ de $V$ ([Khlif, 2000], p.26). C'est une conséquence du fait suivant : si $x_{1}, x_{2}, x_{3}, x_{4}$ sont quatre points de $M$, il existe une transformation conforme $g$ telle que les transformés $g \cdot x_{1}, g \cdot x_{2}, g \cdot x_{3}, g \cdot x_{4}$ appartiennent à $V$. L'invariance du birapport caractérise le groupe conforme : un difféomorphisme local 
de $V$ préservant le birapport se prolonge en une transformation conforme ([Kantor, 1967], Theorem 6).

4. Algèbre de Jordan euclidienne involutive. Soient $V$ une algèbre de Jordan euclidienne et $\alpha$ un automorphisme involutif de $V$. Nous dirons que la paire $(V, \alpha)$ est une algèbre de Jordan euclidienne involutive. Les sous-espaces propres de $\alpha$

$$
V_{0}=\{x \in V \mid \alpha(x)=x\}, \quad V_{1}=\{x \in V \mid \alpha(x)=-x\}
$$

sont orthogonaux, et $V_{0}$ est une sous-algèbre de Jordan. L'algèbre de Jordan involutive $(V, \alpha)$ est dite irréductible si elle n'admet pas de décomposition non triviale

$$
(V, \alpha) \simeq\left(V^{\prime} \oplus V^{\prime \prime}, \alpha^{\prime} \oplus \alpha^{\prime \prime}\right) .
$$

On montre qu'alors soit $V$ est simple, soit $(V, \alpha) \simeq(W \oplus W, \beta)$, où $W$ est une algèbre de Jordan euclidienne simple et $\beta(u, v)=(v, u)$.

On notera $r_{0}$ le rang de $V_{0}$, et généralement l'indice 0 indiquera une relation à $V_{0}$, et l'indice 1 à $V_{1}$. En particulier $\Omega_{0}$ désignera le cône symétrique de l'algèbre euclidienne $V_{0}$ : $\Omega_{0}=V_{0} \cap \Omega$. Suivant [Kayoya,1994] nous classons les algèbres de Jordan euclidiennes involutives irréductibles en quatre types.

Type I: L'algèbre $V$ est simple, et $V_{0}$ n'est pas simple. Il existe dans ce cas un idempotent $c(c \neq 0, e)$ de $V$ tel que

$$
\alpha(x)=P(w) x
$$

où $w=e-2 c$, et alors

$$
V_{0}=V(c, 0) \oplus V(c, 1), \quad V_{1}=V\left(c, \frac{1}{2}\right) .
$$

(Les valeurs propres de $L(c)$ sont les nombres $0, \frac{1}{2}$ et1, et $V(c, 0), V\left(c, \frac{1}{2}\right)$ et $V(c, 1)$ désignent les sous-espaces propres correspondants.)

EXEMPLE. $V=\operatorname{Sym}(m, \mathbb{R}), \alpha(x)=I_{p q} x I_{p q}$, où

$$
I_{p q}=\left(\begin{array}{cc}
I_{p} & 0 \\
0 & -I_{q}
\end{array}\right) \quad(p+q=m) .
$$

Type II: Les algèbres $V$ et $V_{0}$ sont simples et $r=r_{0}$.

Exemple. $V=\operatorname{Herm}(m, \mathbb{C}), \alpha(x)=\bar{x}=x^{T}$, et alors

$$
V_{0}=\operatorname{Sym}(m, \mathbb{R}), \quad V_{1}=i \operatorname{Skew}(m, \mathbb{R}) .
$$

Type III: Les algèbres $V$ et $V_{0}$ sont simples et $r=2 r_{0}$.

Exemple. $V=\operatorname{Sym}(2 \ell, \mathbb{R}), \alpha(x)=J x J^{-1}$, où

$$
J=\left(\begin{array}{cc}
0 & I_{\ell} \\
-I_{\ell} & 0
\end{array}\right),
$$

et alors

$$
V_{0} \simeq \operatorname{Herm}(\ell, \mathbb{C}), \quad V_{1} \simeq \operatorname{Sym}(\ell, \mathbb{C}) .
$$

Type $I V$ : L'algèbre $V$ n'est pas simple. Dans ce cas

$$
V=W \oplus W, \quad \alpha(u, v)=(v, u),
$$


où $W$ est une algèbre de Jordan euclidienne simple, et alors

$$
V_{0}=\{(u, u) \mid u \in W\} \simeq W, \quad V_{1}=\{(u,-u) \mid u \in W\} .
$$

Nous étendons $\alpha$ en un automorphisme $\mathbb{C}$-linéaire de l'algèbre de Jordan complexifiée $V_{\mathbb{C}}$. Le sous-espace $V^{d}=V_{0}+i V_{1} \subset V_{\mathbb{C}}$ est une algèbre de Jordan réelle semi-simple (non euclidienne). Nous dirons que $\left(V^{d}, \alpha\right)$ est l'algèbre de Jordan involutive duale de $(V, \alpha)$. La forme bilinéaire définie sur $V^{d}$ par

$$
\sigma(x, y)=\operatorname{tr}(x \alpha(y))
$$

est définie positive. En effet, si $x=x_{0}+i x_{1}\left(x_{0} \in V_{0}, x_{1} \in V_{1}\right)$,

$$
\sigma(x, x)=\left\|x_{0}\right\|^{2}+\left\|x_{1}\right\|^{2} .
$$

Réciproquement on montre que toute algèbre de Jordan réelle semi-simple involutive $(W, \alpha)$, pour laquelle la forme bilinéaire

$$
\sigma(x, y)=\operatorname{tr}(x \alpha(y))
$$

est définie positive, est obtenue de cette façon.

Remarquons que dans le cas d'une algèbre de Jordan euclidienne involutive $(V, \alpha)$ de type IV l'algèbre $V^{d}$ est isomorphe à l'algèbre de Jordan complexe $\left(V_{0}\right)_{\mathbb{C}}$ considérée comme une algèbre réelle.

Nous terminons cette section en présentant deux formules d'intégration qui seront utilisées en analyses de Fourier et Laplace sphériques dans les sections 6 et 7 . Nous supposons que l'algèbre de Jordan euclidienne involutive $(V, \alpha)$ est irréductible, et que $V_{0}$ est simple.

Proposition 4.1. (a) Pour $\Re \nu>\frac{n}{r}-\frac{r_{0}}{r}$,

$$
\int_{V_{1}} \Delta\left(e+i x_{1}\right)^{-\nu} d x_{1}=(2 \pi)^{n_{1}} \frac{\Gamma_{\Omega_{0}}\left(\frac{r}{r_{0}} \nu-\frac{n_{1}}{r_{0}}\right)}{\Gamma_{\Omega}(\nu)} .
$$

(b) Pour $x_{0} \in \Omega_{0}$,

$$
\int_{V_{1}} \Delta\left(x_{0}+x_{1}\right)^{-\nu} d x_{1}=(2 \pi)^{n_{1}} \frac{\Gamma_{\Omega_{0}}\left(\frac{r}{r_{0}} \nu-\frac{n_{1}}{r_{0}}\right)}{\Gamma_{\Omega}(\nu)} \Delta\left(x_{0}\right)^{\frac{n_{1}}{r}-\nu} .
$$

[van Dijk-Pevzner, 2001], démonstration du Theorem 10.1.

Démonstration. (a) D'après [Faraut-Korányi, 1994], Corollary VII.1.3,

$$
\Delta\left(e+i x_{1}\right)^{-\nu}=\frac{1}{\Gamma_{\Omega}(\nu)} \int_{\Omega} e^{-\left(e+i x_{1} \mid y\right)} \Delta(y)^{\nu-\frac{n}{r}} d y
$$

et nous pouvons écrire

$$
\Delta\left(e+i x_{1}\right)^{-\nu}=\frac{1}{\Gamma_{\Omega}(\nu)} \int_{V_{1}} e^{-i\left(x_{1} \mid y_{1}\right)} h\left(y_{1}\right) d y_{1} .
$$

où $h$ est la fonction définie sur $V_{1}$ par

$$
h\left(y_{1}\right)=\int_{\left\{y_{0} \in V_{0} \mid y_{0}+y_{1} \in \Omega\right\}} e^{-\operatorname{tr}\left(y_{0}\right)} \Delta\left(y_{0}+y_{1}\right)^{\nu-\frac{n}{r}} d y_{0} .
$$


D'après la formule d'inversion de la transformation de Fourier

$$
\begin{aligned}
\int_{V_{1}} \Delta\left(e+i x_{1}\right)^{\nu} d x_{1} & =\frac{(2 \pi)^{n_{1}}}{\Gamma_{\Omega}(\nu)} h(0) \\
& =\frac{(2 \pi)^{n_{1}}}{\Gamma_{\Omega}(\alpha)} \int_{\Omega_{0}} e^{-\operatorname{tr}\left(y_{0}\right)} \Delta\left(y_{0}\right)^{\nu-\frac{n_{1}}{r}-\frac{n_{0}}{r}} d y_{0} \\
& =(2 \pi)^{n_{1}} \frac{\Gamma_{\Omega_{0}}\left(\frac{r}{r_{0}} \nu-\frac{n_{1}}{r_{0}}\right)}{\Gamma_{\Omega}(\nu)} .
\end{aligned}
$$

(b) On pose $x_{1}=P\left(x_{0}^{\frac{1}{2}}\right) u_{1}$, alors $d x_{1}=\Delta\left(x_{0}\right)^{\frac{n_{1}}{r}} d u_{1}$, et

$$
\int_{V_{1}} \Delta\left(x_{0}+x_{1}\right)^{-\nu} d x_{1}=\Delta\left(x_{0}\right)^{\frac{n_{1}}{r}-\nu} \int_{V_{1}} \Delta\left(e+u_{1}\right)^{-\nu} d u_{1} .
$$

Proposition 4.2. (a) Pour $\Re \nu>\frac{n}{r}-1$,

$$
\int_{\left\{x_{1} \in V_{1} \mid e+x_{1} \in \Omega\right\}} \Delta\left(e+x_{1}\right)^{\nu-\frac{n}{r}} d x_{1}=\frac{\Gamma_{\Omega}(\nu)}{\Gamma_{\Omega_{0}}\left(\frac{r}{r_{0}} \nu\right)} .
$$

(b) Pour $x_{0} \in \Omega_{0}$,

$$
\int_{\left\{x_{1} \in V_{1} \mid x_{0}+x_{1} \in \Omega\right\}} \Delta\left(x_{0}+x_{1}\right)^{\nu-\frac{n}{r}} d x_{1}=\frac{\Gamma_{\Omega}(\nu)}{\Gamma_{\Omega_{0}}\left(\frac{r}{r_{0}} \nu\right)} \Delta\left(x_{0}\right)^{\nu-\frac{n_{0}}{r}} .
$$

[Ben Saïd, 2000], Lemma 5.4.

Démonstration. (a) Notons $I(\nu)$ la valeur de la première intégrale, et calculons l'intégrale

$$
\Gamma_{\Omega}(\nu)=\int_{\Omega} e^{-\operatorname{tr}(x)} \Delta(x)^{\nu-\frac{n}{r}} d x
$$

par intégrations successives :

$$
=\int_{\Omega_{0}} e^{-\operatorname{tr}\left(x_{0}\right)}\left(\int_{\left\{x_{1} \in V_{1} \mid x_{0}+x_{1} \in \Omega\right\}} \Delta\left(x_{0}+x_{1}\right)^{\nu-\frac{n}{r}} d x_{1}\right) d x_{0} .
$$

En posant $x_{1}=P\left(x_{0}^{\frac{1}{2}}\right) u_{1}, d x_{1}=\Delta\left(x_{0}\right)^{\frac{n_{1}}{r}} d u_{1}$, nous obtenons

$$
\begin{aligned}
\Gamma_{\Omega}(\nu) & =I(\nu) \int_{\Omega_{0}} e^{-\operatorname{tr}\left(x_{0}\right)} \Delta\left(x_{0}\right)^{\nu-\frac{n_{0}}{r}} d x_{0} \\
& =I(\nu) \Gamma_{\Omega_{0}}\left(\frac{r}{r_{0}} \nu\right) .
\end{aligned}
$$

(b) La démonstration est analogue à celle de la partie (b) de la proposition 4.1.

5. Espace symétrique ordonné de Makarevic. Soit $V$ une algèbre de Jordan euclidienne, et soit $\varphi: U_{1} \rightarrow U_{2}$ un difféomorphisme local $\left(U_{1}\right.$ et $U_{2}$ sont deux ouverts de $V)$. La transformation $\varphi$ est dite causale si, pour tout $x$ de $U_{1}$, sa différentielle $(D \varphi)_{x}$ appartient à $G(\Omega)$. Clairement les translations et les transformations linéaires de $G(\Omega)$ sont causales. La transformation $j: x \mapsto-x^{-1}$ est aussi causale car $(D j)_{x}=P\left(x^{-1}\right)$, et, pour $x$ inversible, $P(x)$ appartient à $G(\Omega)$. Nous appelerons groupe causal de $V$, et note- 
rons Caus $(V)$, le groupe des transformations rationnelles engendré par les translations, $G(\Omega)$ et la transformation $j$. Du fait que

$$
\operatorname{Str}(V)=G(\Omega) \times\{ \pm I\},
$$

il résulte que Caus $(V)$ est un sous-groupe d'ordre 2 du groupe conforme $\operatorname{Conf}(V)$, et il opère transitivement sur la compactification conforme $M$ de $V$. Par suite la variété $M$ possède une structure causale dont la restriction à $V$ est la structure causale plate définie par le cône $-\bar{\Omega}$, et qui est invariante $\operatorname{par} \operatorname{Caus}(V)$.

Soit $\alpha$ un automorphisme involutif de $V$. Suivant la construction de [Bertram, 1996b, 1998] on associe à l'algèbre de Jordan euclidienne involutive $(V, \alpha)$ un espace symétrique ordonné, qui est appelé espace symétrique ordonné de Makarevic. On pose

$$
G=\{g \in \operatorname{Caus}(V) \mid(-\alpha) \circ g \circ(-\alpha)=g\}_{0} .
$$

Alors l'orbite $\mathcal{M}=G \cdot e$ est un ouvert de la compactification conforme $M$ de $V, \mathcal{M}=G / H$ où

$$
H=\{g \in G \mid g \cdot e=e\} .
$$

L'espace homogène $\mathcal{M}=G / H$ est symétrique relativement à l'involution de $G$ définie par

$$
\sigma(g)=(-j) \circ g \circ(-j) .
$$

Il est muni de la structure causale invariante induite par celle de $M$, et on montre qu'il est un espace symétrique ordonné. En général $\mathcal{M}$ n'est pas contenu dans $V$, c'est à dire que $\mathcal{M}$ contient des points "à l'infini". De plus tous les points de $V$ n'appartiennent pas à $\mathcal{M}$. On montre que

$$
\mathcal{M} \cap V \subset\{x \in V \mid \Delta(x+\alpha(x)) \neq 0\}
$$

([Bertram, 1998], Theorem 2.1.3). Avec le choix de la structure causale pour laquelle $C_{x}=-\bar{\Omega}$, on montre que

$$
\mathcal{M}^{+}:=\{x \in \mathcal{M} \mid x \geq e\} \subset V
$$

Plus précisément

$$
\mathcal{M}^{+}=(e-\bar{\Omega}) \cap\left\{x_{0} \in \Omega_{0}\right\}
$$

([Ben Saïd, 2000], Proposition 5.2).

Si l'algèbre involutive $(V, \alpha)$ est de type IV, $\mathcal{M}$ est un espace symétrique de type Cayley.

L'algèbre de Lie $\mathfrak{g}$ de $G$ est composée des champs de vecteurs de la forme

$$
\xi(x)=u+T x-P(x) v,
$$

avec $u, v \in V_{1}$, et $T \in \mathfrak{s t r}(V)$ vérifie $\alpha \circ T \circ \alpha=T$. Ceux qui composent

$$
\mathfrak{h}=\operatorname{Lie}(H)=\{\xi \in \mathfrak{g} \mid d \sigma(\xi)=\xi\}
$$

vérifient de plus $u=v, T^{*}=-T$, et ceux qui composent

$$
\mathfrak{q}=\{\xi \in \mathfrak{g} \mid d \sigma(\xi)=-\xi\}
$$

vérifient $u=-v, T^{*}=T$. L'involution $\theta$ de $G$ définie par

$$
\theta(g)=j \circ g \circ j
$$


est une involution de Cartan qui commute à $\sigma$, et $K=\{g \in G \mid \theta(g)=g\}$ est un sous-groupe compact maximal de $G$. Notons aussi que

$$
\begin{aligned}
\mathfrak{k} & =\operatorname{Lie}(K)=\{\xi \in \mathfrak{g} \mid d \theta(\xi)=\xi\} \\
& =\left\{\xi(x)=u+T x-P(x) v \mid v=-u, T^{*}=-T\right\}, \\
\mathfrak{p} & =\{\xi \in \mathfrak{g} \mid d \theta(\xi)=-\xi\} \\
& =\left\{\xi(x)=u+T x-P(x) v \mid v=u, T^{*}=T\right\} .
\end{aligned}
$$

L'algèbre de Lie est graduée, et sa graduation est définie par $\operatorname{ad}\left(\xi_{0}\right)$, où $\xi_{0}(x)=x$ :

$$
\mathfrak{g}=\mathfrak{g}_{1} \oplus \mathfrak{g}_{0} \oplus \mathfrak{g}_{-1},
$$

avec

$$
\begin{aligned}
\mathfrak{g}_{1} & =\left\{\xi(x)=u \mid u \in V_{1}\right\}, \\
\mathfrak{g}_{0} & =\{\xi(x)=T x \mid T \in \mathfrak{s t r}(V), \alpha \circ T \circ \alpha=T\}, \\
\mathfrak{g}_{-1} & =\left\{\xi(x)=-P(x) v \mid v \in V_{1}\right\} .
\end{aligned}
$$

Dans toute la suite nous excluerons le type I : nous supposons que l'automorphisme $\alpha$ n'est pas trivial, et que $(V, \alpha)$ est de type II, III ou IV. Soit $\left\{c_{1}, \ldots, c_{r_{0}}\right\}$ un repère de Jordan de $V_{0}$. Le sous-espace $\mathfrak{a}$ de $\mathfrak{g}$ défini par

$$
\mathfrak{a}=\left\{\xi(x)=\sum_{j=1}^{r_{0}} t_{j} L\left(c_{j}\right) \mid t_{j} \in \mathbb{R}\right\}
$$

est un sous-espace de Cartan contenu dans $\mathfrak{p} \cap \mathfrak{q}$. On pose $A=\exp \mathfrak{a}$. La condition $0<t_{1}<\cdots<t_{r_{0}}$ définit une chambre de Weyl $\mathfrak{a}^{+}$pour le système de racines (restreintes) $\Delta(\mathfrak{g}, \mathfrak{a})$. On note $\Delta^{+}$le système positif correspondant. Il se décompose en $\Delta^{+}=\Delta_{0}^{+} \cup \Delta_{1}$ où $\Delta_{0}^{+}$est un système positif dans $\Delta_{0}=\Delta\left(\mathfrak{g}_{0}, \mathfrak{a}\right)$, et $\Delta_{1}$ est l'ensemble des racines $\beta$ pour lesquelles $\mathfrak{g}^{\beta} \subset \mathfrak{g}_{1}$. On pose

$$
\begin{aligned}
& \mathfrak{n}_{0}=\bigoplus_{\beta \in \Delta_{0}^{+}} \mathfrak{g}^{\beta}, \quad N_{0}=\exp \mathfrak{n}_{0}, \\
& \mathfrak{n}_{1}=\mathfrak{g}_{1}=\bigoplus_{\beta \in \Delta_{1}} \mathfrak{g}^{\beta}, \quad N_{1}=\exp \mathfrak{n}_{1},
\end{aligned}
$$

et $N=N_{0} \ltimes N_{1}$. Une transformation $n$ de $N$ s'écrit

$$
n \cdot x=n_{0} \cdot x+v_{1},
$$

où $n_{0}$ appartient au groupe triangulaire stricte $N_{0}$ du cône symétrique $\Omega_{0}, N_{0} \subset G\left(\Omega_{0}\right)$, et $v_{1} \in V_{1}$.

Soit $\rho$ la demi-somme des racines de $\Delta^{+}, \rho=\rho_{0}+\rho_{1}$, où $\rho_{0}$ est la demi-somme des racines de $\Delta_{0}^{+}$et $\rho_{1}$ celle des racines de $\Delta_{1}$. Si

$$
\xi(x)=\sum_{j=1}^{r_{0}} t_{j} L\left(c_{j}\right) x,
$$

alors

$$
\rho_{0}(\xi)=\sum_{j=1}^{r_{0}} \frac{d_{0}}{4}\left(2 j-r_{0}-1\right) t_{j}, \quad \rho_{1}(\xi)=\frac{1}{2} \frac{n_{1}}{r_{0}} \sum_{j=1}^{r_{0}} t_{j} .
$$




\begin{tabular}{c|cccc}
$(V, \alpha)$ & Type I & Type II & Type III & Type IV \\
\hline$\Delta(\mathfrak{g}, \mathfrak{a})$ & $A_{r-1}$ & $D_{r}$ & $C_{r_{0}}$ & $C_{r_{0}}$
\end{tabular}

Sur l'espace $\mathcal{M}$ nous nous intéressons à l'analyse du noyau $F$ défini par

$$
F(x, y)=\{x,-\alpha(y), y,-\alpha(x)\}=\frac{\Delta(x-y)^{2}}{\Delta(x+\alpha(x)) \Delta(y+\alpha(y))} .
$$

Il est invariant par $G$, et si $x \geq y, F(x, y) \geq 0$. Nous pouvons donc considérer ses puissances $F^{\nu}(x, y)(\nu \in \mathbb{C})$ qui nous permettrons de définir les intégrales de Riesz. Pour les étudier nous utiliserons certains résultats d'analyse harmonique sur l'espace riemannien dual $\mathcal{M}^{d}$ que nous allons présenter dans la section suivante.

6. Espace symétrique riemannien dual. Nous allons décrire l'espace riemannien symétrique $\mathcal{M}^{d}$ dual (au sens de Flensted-Jensen) de l'espace symétrique ordonné $\mathcal{M}$, associé à une algèbre de Jordan euclidienne involutive $(V, \alpha)$ irréductible. Rappelons que l'algèbre de Jordan duale $V^{d}$ est définie par $V^{d}=V_{0}+i V_{1}$. Soit $G^{d}$ le sous-groupe de $\operatorname{Conf}\left(V^{d}\right)$ défini par

$$
G^{d}=\left\{g \in \operatorname{Conf}\left(V^{d}\right) \mid(-\alpha) \circ g \circ(-\alpha)=g\right\}_{0} .
$$

L'orbite $\mathcal{M}^{d}=G^{d} \cdot e$ est un ouvert de $V$ qui est un domaine tubulaire

$$
\mathcal{M}^{d}=\Omega_{0}+i V_{1} \text {. }
$$

Le sous-groupe d'isotropie $K^{d}$ de $e$,

$$
K^{d}=\left\{g \in G^{d} \mid g \cdot e=e\right\},
$$

est compact et $\mathcal{M}^{d}=G^{d} / K^{d}$ est un espace symétrique riemannien. Si l'algèbre involutive $(V, \alpha)$ est de type IV, $V^{d} \simeq\left(V_{0}\right)_{\mathbb{C}}$, et $\mathcal{M}^{d}$ est un domaine tubulaire hermitien symétrique

$$
\mathcal{M}^{d}=\Omega_{0}+i V_{0} .
$$

L'algèbre de Lie de $G^{d}$ est égale à

$$
\mathfrak{g}^{d}=\mathfrak{k} \cap \mathfrak{h} \oplus \mathfrak{p} \cap \mathfrak{q} \oplus i \mathfrak{k} \cap \mathfrak{q} \oplus i \mathfrak{p} \cap \mathfrak{h},
$$

et celle de $K^{d}$ à

$$
\mathfrak{k}^{d}=\mathfrak{k} \cap \mathfrak{h}+i \mathfrak{p} \cap \mathfrak{h} .
$$

En fait les groupes $G$ et $G^{d}$ sont conjugués dans $G_{\mathbb{C}}$ :

$$
G^{d}=\gamma \circ G \circ \gamma^{-1}, \quad \mathfrak{g}^{d}=\operatorname{Ad}(\gamma) \mathfrak{g},
$$

où $\gamma$ est l'élément de $G_{\mathbb{C}}$ défini par $\gamma \cdot x=i x$. De plus

$$
K^{d}=\gamma \circ K \circ \gamma^{-1}, \quad \mathfrak{k}^{d}=\operatorname{Ad}(\gamma) \mathfrak{k} .
$$

La décomposition d'Iwasawa de $G^{d}=N^{d} A K^{d}$ peut être décrite comme suit : tout $x$ de $\mathcal{M}^{d}$ s'écrit de façon unique

$$
x=n a \cdot e=n_{0} a \cdot e+i v_{1},
$$

où $a \in A, n_{0} \in N_{0}$ et $v_{1} \in V_{1}$, et on note

$$
a=\exp \mathcal{A}(x), \quad \text { avec } \mathcal{A}(x) \in \mathfrak{a} .
$$


Si $\lambda \in \mathfrak{a}_{\mathbb{C}}^{*}$, on pose, si $\xi(x)=\sum t_{j} L\left(c_{j}\right)$,

$$
\lambda(\xi)=\sum_{j=1}^{r_{0}} \lambda_{j} t_{j} \quad\left(\lambda_{j} \in \mathbb{C}\right),
$$

et alors

$$
e^{\langle\lambda, \mathcal{A}(x)\rangle}=\Delta_{\lambda}^{0}(x),
$$

où $\Delta_{\lambda}^{0}$ est la fonction puissance du cône symétrique $\Omega_{0}$.

La transformée de Fourier sphérique d'une fonction $f$ définie sur $\mathcal{M}^{d}$ et invariante par $K^{d}$ peut s'écrire

$$
\begin{aligned}
\mathcal{F} f(\lambda) & =\int_{\mathcal{M}^{d}} e^{\langle\rho-\lambda, \mathcal{A}(x)\rangle} f(x) d m(x) \\
& =\int_{\Omega_{0} \times V_{1}} \Delta_{\rho-\lambda}^{0} f\left(x_{0}+i x_{1}\right) \Delta\left(x_{0}\right)^{-\frac{n}{r}} d x_{0} d x_{1} .
\end{aligned}
$$

La mesure $d m(x)=\Delta\left(x_{0}\right)^{-\frac{n}{r}} d x_{0} d x_{1}$ est invariante par $G$.

Soit $F^{d}$ le noyau défini sur $\mathcal{M}^{d}$ par

$$
F^{d}(x, y)=\{x, y,-\alpha(x),-\alpha(y)\}=\frac{\Delta(x+\alpha(x)) \Delta(y+\alpha(y))}{\Delta(y+\alpha(x)) \Delta(x+\alpha(y))} .
$$

C'est un noyau invariant par $G^{d}$ qui est positif. Pour $\nu \in \mathbb{C}$ nous posons

$$
B_{\nu}(x, y)= \begin{cases}F^{d}(x, y)^{\nu} & \text { si }(V, \alpha) \text { est de type II, } \\ F^{d}(x, y)^{\frac{\nu}{2}} & \text { si }(V, \alpha) \text { est de type III ou IV. }\end{cases}
$$

C'est le noyau de Berezin. Soit $\psi_{\nu}$ la fonction $K^{d}$-invariante qui lui est associée

$$
\psi_{\nu}(x)=B_{\nu}(x, e) .
$$

Si $(V, \alpha)$ est de type II,

$$
\psi_{\nu}(x)=\frac{\Delta\left(\frac{x+e}{2}\right)^{-2 \nu}}{\Delta\left(x_{0}\right)^{-\nu}} \quad\left(x=x_{0}+i x_{1}\right)
$$

et, si $x=\sum_{j=1}^{r_{0}} e^{t_{j}} c_{j}$,

$$
\psi_{\nu}(x)=\prod_{j=1}^{r_{0}}\left(\operatorname{ch} \frac{t_{j}}{2}\right)^{-2 \nu} .
$$

Sa transformée de Fourier sphérique a été calculée par van Dijk et Pevzner ([2001]). Elle avait été calculée par Unterberger et Upmeier ([1994]) dans le cas où $(V, \alpha)$ est de type IV.

ThÉORÈme 6.1. Supposons $\Re \nu>\delta-\frac{1}{2}$. Pour $\left|\Re \lambda_{j}\right|<\Re \nu-\delta+\frac{1}{2}$, la transformée de Fourier sphérique de $\psi_{\nu}$ est égale à

$$
\mathcal{F} \psi_{\nu}(\lambda)=\frac{1}{\beta(\nu)} \prod_{j=1}^{r_{0}} \Gamma\left(\frac{1}{2}+\nu-\delta+\lambda_{j}\right) \Gamma\left(\frac{1}{2}+\nu-\delta-\lambda_{j}\right),
$$

ò̀ $\delta=\frac{n}{2 r_{0}}$ et

$$
\beta(\nu)= \begin{cases}c 2^{-2 r \nu} \Gamma_{\Omega}(2 \nu) & \text { si }(V, \alpha) \text { est de type II, } \\ c 2^{-r \nu} \Gamma_{\Omega}(\nu) & \text { si }(V, \alpha) \text { est de type III ou IV. }\end{cases}
$$


Démonstration. Nous en donnons la démonstration dans le cas où $(V, \alpha)$ est de type II. En utilisant la formule (2) de la section 2 nous obtenons

$$
\begin{aligned}
& \mathcal{F} \psi_{\nu}(\lambda) \\
& =2^{2 r \nu} \int_{\Omega_{0} \times V_{1}} \Delta_{\rho-\lambda+\nu-\frac{n_{1}}{r}}^{0}\left(x_{0}\right) \Delta\left(e+x_{0}+i x_{1}\right)^{-2 \nu} \Delta\left(x_{0}\right)^{-\frac{n_{0}}{r}} d x_{0} d x_{1} .
\end{aligned}
$$

Calculant d'abord l'intégrale en $x_{1}$ en utilisant la proposition 4.1, nous obtenons

$$
\begin{aligned}
& \mathcal{F} \psi_{\nu}(\lambda)=2^{2 r \nu}(2 \pi)^{n_{1}} \frac{\Gamma_{\Omega_{0}}\left(2 \nu-\frac{n_{1}}{r}\right)}{\Gamma_{\Omega}(2 \nu)} \\
& \int_{\Omega_{0}} \Delta_{\rho_{0}-\lambda+\nu-\frac{n_{1}}{2 r}}^{0}\left(x_{0}\right) \Delta\left(e+x_{0}\right)^{\frac{n_{1}}{r}-2 \nu} \Delta\left(x_{0}\right)^{-\frac{n_{0}}{r}} d x_{0} .
\end{aligned}
$$

Puis en utilisant la formule (2) de la section 2 nous obtenons

$$
\mathcal{F} \psi_{\nu}(\lambda)=(2 \pi)^{n_{1}} 2^{2 r \nu} \frac{\Gamma_{\Omega_{0}}\left(\rho+\lambda+\nu-\frac{n_{1}}{r}\right) \Gamma_{\Omega_{0}}\left(\rho-\lambda+\nu-\frac{n_{1}}{r}\right)}{\Gamma_{\Omega}(2 \nu)} .
$$

Le résultat s'en déduit car

$$
\Gamma_{\Omega_{0}}\left(\rho_{0}+\mu\right)=c \prod_{j=1}^{r_{0}} \Gamma\left(\frac{1}{2}-\frac{n_{0}}{2 r_{0}}+\mu_{j}\right)
$$

De ce calcul de transformée de Fourier sphérique on déduit une identité de type Bernstein remarquable. Rappelons d'abord que l'homomorphisme de Harish-Chandra $\gamma$ associe à tout opérateur différentiel invariant $D \in \mathbb{D}\left(G^{d} / K^{d}\right)$ un polynôme invariant par le groupe de Weyl $W$, et que l'application $D \mapsto \gamma_{D}$ est un isomorphisme d'algèbre de $\mathbb{D}\left(G^{d} / K^{d}\right)$ sur $S\left(\mathfrak{a}_{\mathbb{C}}\right)^{W}$. Dans le cas que nous considérons $W=\mathfrak{S}_{r} \ltimes\{1,-1\}^{r_{0}}$. Rappelons que, si $f$ est une fonction $K^{d}$-invariante sur $\mathcal{M}^{d}$, la transformée de Fourier de $D f$ est égale à $\gamma_{D}(\lambda) \mathcal{F} f(\lambda)$.

Le polynôme $\gamma_{\nu}$ défini par

$$
\gamma_{\nu}(\lambda)=\prod_{j=1}^{r_{0}}\left(\left(\nu-\frac{1}{2}\right)^{2}-\lambda_{j}^{2}\right)
$$

est invariant par $W$, et, par conséquent, il existe un opérateur différentiel invariant $D(\nu)$ tel que

$$
\gamma_{D(\nu)}=\gamma_{\nu}
$$

Corollaire 6.2.

$$
D(\delta-\nu) B_{\nu}(x, y)=b(\nu) B_{\nu+1}(x, y)
$$

l'opérateur $D(\delta-\nu)$ agissant sur la variable $x ; b(\nu)$ est un polynôme de degré $2 r$, et $\delta=\frac{n}{2 r_{0}}$.

Dans le cas où $(V, \alpha)$ est de type IV, cette identité a été établie par Engliš ([1999], Proposition 4). Elle est aussi énoncée de façon un peu différente dans [UnterbergerUpmeier, 1994]. 
Démonstration. En utilisant la relation $\Gamma(z+1)=z \Gamma(z)$ nous déduisons du théorème 6.1 que,

$$
\frac{\mathcal{F} \psi_{\nu+1}(\lambda)}{\mathcal{F} \psi_{\nu}(\lambda)}=\frac{1}{b(\nu)} \prod_{j=1}^{r_{0}}\left(\nu-\delta+\frac{1}{2}+\lambda_{j}\right)\left(\nu-\delta+\frac{1}{2}-\lambda_{j}\right)=\frac{1}{b(\nu)} \gamma_{\delta-\nu}(\lambda),
$$

où

$$
b(\nu)=\frac{\beta(\nu+1)}{\beta(\nu)}
$$

7. Intégrales de Riesz. Sur l'espace symétrique ordonné de Makarevic $\mathcal{M}$ qui a été introduit dans la section 5 , associé à l'algèbre de Jordan euclidienne involutive $(V, \alpha)$, considérons le noyau

$$
F(x, y)=\{x,-\alpha(y), y,-\alpha(x)\}=\frac{\Delta(x-y)^{2}}{\Delta(x+\alpha(x)) \Delta(y+\alpha(y))} .
$$

Il est bien défini, car, pour tout point $x$ de $\mathcal{M}, \Delta(x+\alpha(x)) \neq 0$, et il est invariant par $G$ puisque $G \subset \operatorname{Conf}(V)$, et qu'une transformation $g$ de $G$ commute à $(-\alpha)$. Les noyaux $F^{d}$ et $F$ sont reliés par la relation

$$
F(x, e)=F^{d}(x,-e)^{-1},
$$

et, en considérant $F^{\nu}$ comme une fonction multiforme,

$$
F(x, e)^{\nu}=B_{-\nu}(x,-e) .
$$

Notons que

$$
F(x, e)=\frac{\Delta\left(\frac{x-e}{2}\right)^{2}}{\Delta\left(x_{0}\right)}
$$

et que, si

$$
x=\sum_{j=1}^{r_{0}} t_{j} c_{j}
$$

alors

$$
F(x, e)=\prod_{j=1}^{r_{0}}\left(\operatorname{sh} \frac{t_{j}}{2}\right)^{2}
$$

Supposons d'abord que $(V, \alpha)$ soit de type II. Par prolongement analytique on déduit de ce qui précède que, $D(\nu+\delta)$ agissant sur la variable $x$,

$$
D(\nu+\delta) F(x, y)^{\nu}=b(-\nu) F(x, y)^{\nu-1} .
$$

Pour une fonction $\varphi \in \mathcal{C}_{c}^{\infty}(\mathcal{M})$, l'intégrale de Riesz $R_{\nu}(\varphi)$ est définie par

$$
R_{\nu}(\varphi)=\frac{1}{\Gamma_{\Omega}(2 \nu)} \int_{\mathcal{M}^{+}} F(x, e)^{\nu-\delta} \varphi(x) d m(x) .
$$

Cette intégrale converge pour $\Re \nu>\delta-\frac{1}{2}$, et définit une distribution qui est une fonction holomorphe de $\nu$. Si $(V, \alpha)$ est de type III ou IV nous posons

$$
R_{\nu}(\varphi)=\frac{1}{\Gamma_{\Omega}(\nu)} \int_{\mathcal{M}^{+}} F(x, e)^{\frac{\nu-\delta}{2}} \varphi(x) d m(x) .
$$


ThÉORÈme 7.1. Pour $\Re \nu$ assez grand,

$$
D(\nu) R_{\nu}=R_{\nu-1}
$$

Par suite, comme fonction de $\nu, R_{\nu}$ admet un prolongement holomorphe qui est une fonction entière de $\nu$.

Si $(V, \alpha)$ est de type IV ce résultat est du à Khlif ([2000], théorème 3.4.1 et corollaire 3.4.3).

Pour $\nu=0$,

$$
R_{0}(\varphi)=\varphi(e)
$$

Par suite $R_{1}$ est une solution élémentaire de $D(1)$,

$$
D(1) R_{1}=\delta_{e} .
$$

Plus généralement, pour $m \in \mathbb{N}^{*}$, l'opérateur $D(m)$ admet pour solution élémentaire

$$
E_{m}=D(m-1) \ldots D(1) R_{m} .
$$

Nous allons calculer la transformée de Laplace sphérique de $R_{\nu}$. Observons que $N A$. $e=\Omega_{0}+V_{1}$, et que, conformément à la théorie générale,

$$
\mathcal{M}^{+} \subset N A \cdot e \text {. }
$$

Si $x=n \exp X \cdot e$, on pose $\mathcal{A}(x)=X$. Ainsi $x \mapsto \mathcal{A}(x)$ est une application à valeurs dans a définie sur $N A \cdot e=\Omega_{0}+V_{1}$. La transformée de Laplace sphérique d'un noyau de Volterra $K$ peut s'écrire :

$$
\begin{aligned}
\mathcal{L} K(\lambda) & =\int_{\mathcal{M}^{+}} e^{\langle\rho-\lambda, \mathcal{A}(x)\rangle} K(x, e) d m(x) \\
& =\int_{(e-\Omega) \cap\left\{x_{0} \in \Omega_{0}\right\}} \Delta_{\rho-\lambda}^{0}\left(x_{0}\right) F\left(x_{0}+x_{1}, e\right) \Delta\left(x_{0}\right)^{-\frac{n}{r}} d x_{0} d x_{1} .
\end{aligned}
$$

Nous notons $R_{\nu}$ le noyau de Volterra défini par

$$
R_{\nu}(x, y)= \begin{cases}\frac{1}{\Gamma_{\Omega}(2 \nu)} F(x, y)^{\nu-\delta} & \text { si } x \geq y \\ 0 & \text { sinon. }\end{cases}
$$

ThÉORÈme 7.2. Pour $\Re \lambda_{j}<\frac{1}{2}-\Re \nu$, la transformée de Laplace sphérique du noyau de Volterra $R_{\nu}$ est égale à

$$
\mathcal{L} R_{\nu}(\lambda)=\prod_{j=1}^{r_{0}} \frac{\Gamma\left(\frac{1}{2}-\nu-\lambda_{j}\right)}{\Gamma\left(\frac{1}{2}+\nu-\lambda_{j}\right)}
$$

[Ben Saïd, 2000], Proposition 5.5. Ce résultat avait été obtenu par Khlif dans le cas où l'algèbre de Jordan involutive $(V, \alpha)$ est de type IV ([2000], Proposition 3.4.4).

Notons que l'on vérifie bien que

$$
\gamma_{\nu}(\lambda) \mathcal{L} R_{\nu}(\lambda)=\mathcal{L} R_{\nu-1}(\lambda) .
$$

Démonstration. Nous en donnons la démonstration dans le cas de type II. Nous pouvons écrire

$$
\mathcal{L} R_{\nu}(\lambda)=\frac{1}{\Gamma_{\Omega}(2 \nu)} \int_{\mathcal{M}^{+}} \Delta_{\rho-\lambda}^{0}\left(x_{0}\right)\left(\frac{\Delta(x-e)^{2}}{\Delta\left(x_{0}\right)}\right)^{\nu-\frac{n}{2 r}} \Delta\left(x_{0}\right)^{-\frac{n}{r}} d x_{0} d x_{1} .
$$


Nous calculons d'abord l'intégrale en $x_{1}$ en utilisant la proposition 4.2 :

$$
\int_{\left\{x_{1} \in V_{1} \mid e-x_{0}-x_{1} \in \Omega\right\}} \Delta\left(e-x_{0}-x_{1}\right)^{2 \nu-\frac{n}{r}} d x_{1}=\Delta\left(e-x_{0}\right)^{2 \nu-\frac{n_{0}}{r}} \frac{\Gamma_{\Omega}(2 \nu)}{\Gamma_{\Omega_{0}}(2 \nu)} .
$$

L'intégrale en $x_{0}$ se traite en utilisant la formule (a) de la section 2 :

$$
\begin{aligned}
& \int_{\Omega_{0} \cap\left(e-\Omega_{0}\right)} \Delta_{\rho_{0}-\lambda-\nu+\frac{n_{0}}{2 r}}^{0}\left(x_{0}\right) \Delta\left(e-x_{0}\right)^{2 \nu-\frac{n_{0}}{r}} \Delta\left(x_{0}\right)^{-\frac{n_{0}}{r}} d x_{0} \\
& =\frac{\Gamma_{\Omega_{0}}(2 \nu) \Gamma_{\Omega_{0}}\left(\rho-\lambda-\nu+\frac{n_{0}-n_{1}}{2 r}\right)}{\Gamma_{\Omega_{0}}\left(\rho-\lambda+\nu+\frac{n_{0}-n_{1}}{2 r}\right)} .
\end{aligned}
$$

On en déduit le résultat annoncé.

8. L'espace de Lobachevski imaginaire. Dans cette dernière section nous présentons l'exemple le plus simple d'un espace symétrique ordonné de Makarevic. Soit $V=\mathbb{R}^{1, n-1}$ l'algèbre de Jordan pour laquelle le produit est défini par : $z=x \circ y$ si

$$
\begin{aligned}
& z^{0}=x^{0} y^{0}+\cdots+x^{n-1} y^{n-1}, \\
& z^{j}=x^{0} y^{j}+x^{j} y^{0} \quad(j=1, \ldots, n-1) .
\end{aligned}
$$

C'est une algèbre de Jordan euclidienne de rang $r=2$. L'élément neutre est $e=$ $(1,0, \ldots, 0)$, et le déterminant est la forme quadratique de Lorentz

$$
\Delta(x)=\left(x^{0}\right)^{2}-\left(x^{1}\right)^{2}-\cdots-\left(x^{n-1}\right)^{2} .
$$

Le cône symétrique associé est le cône de Lorentz

$$
\Omega=\left\{x \in V \mid \Delta(x)>0, x^{0}>0\right\} .
$$

La composante connexe neutre du groupe causal Caus $(V)$ est un groupe de Lie isomorphe à $S O_{0}(n, 2)$. La compactification conforme $M$ de $V$ peut être réalisée comme la quadratique réelle projective d'équation

$$
\left(y^{0}\right)^{2}-\left(y^{1}\right)^{2}-\cdots-\left(y^{n-1}\right)^{2}-\left(y^{n}\right)^{2}+\left(y^{n+1}\right)^{2}=0,
$$

et le plongement $V \hookrightarrow M$ est défini par

$$
y^{0}=x^{0}, \ldots, y^{n-1}=x^{n-1}, y^{n}=\frac{1+\Delta(x)}{2}, y^{n+1}=\frac{1-\Delta(x)}{2} .
$$

Soit $\alpha$ l'automorphisme de $V$ défini par

$$
\alpha:\left(x^{0}, x^{1}, \ldots, x^{n-1}\right) \mapsto\left(x^{0},-x^{1}, \ldots-x^{n-1}\right) .
$$

Alors

$$
V_{0}=\left\{\left(x^{0}, 0, \ldots, 0\right)\right\} \simeq \mathbb{R}, V_{1}=\left\{\left(0, x^{1}, \ldots x^{n-1}\right)\right\} \simeq \mathbb{R}^{n-1} .
$$

Si $n=2,(V, \alpha)$ est de type IV, et, si $n \geq 3,(V, \alpha)$ est de type III. Dans tous les cas $r_{0}=1$. Le groupe $G$ est isomorphe à $S O_{0}(1, n)$ et $H$ à $S O_{0}(1, n-1)$. L'espace symétrique ordonné de Makarevic $\mathcal{M}$ associé à $(V, \alpha)$ est l'espace de Lobachevski imaginaire. Il est isomorphe à l'hyperbolö̈de d'équation

$$
\left(y^{1}\right)^{2}+\cdots+\left(y^{n}\right)^{2}-\left(y^{n+1}\right)^{2}=1,
$$


l'isomorphisme étant défini sur $\mathcal{M} \cap V$ par

$$
y^{1}=\frac{x^{1}}{x^{0}}, \ldots, y^{n-1}=\frac{x^{n-1}}{x^{0}}, y^{n}=\frac{1+\Delta(x)}{2 x^{0}}, y^{n+1}=\frac{1-\Delta(x)}{2 x^{0}} .
$$

L'isomorphisme inverse est donné par

$$
x_{0}=\frac{1}{y^{n}+y^{n+1}}, x^{1}=\frac{y^{1}}{y^{n}+y^{n+1}}, \ldots, x^{n-1}=\frac{y^{n-1}}{y^{n}+y^{n+1}} .
$$

Pour $y_{1}=\left(y^{1}, \ldots, y^{n+1}\right), y_{2}=\left(y_{2}^{1}, \ldots, y_{2}^{n+1}\right)$, posons

$$
\left[y_{1}, y_{2}\right]=y_{1}^{1} y_{2}^{1}+\cdots+y_{1}^{n} y_{2}^{n}-y_{1}^{n+1} y_{2}^{n+1} .
$$

Dans les variables $y^{j}$ le noyau invariant $F$ défini dans la section 5 s'exprime par

$$
F\left(x_{1}, x_{2}\right)=\frac{1}{4}\left(\left[y_{1}, y_{2}\right]-1\right)^{2} .
$$

L'espace riemannien symétrique dual $\mathcal{M}^{d}$ est l'espace hyperbolique réel de dimension $n$, présenté dans sa réalisation géométrique comme un demi-espace :

$$
\mathcal{M}^{d}=\left\{\left(x^{0}, \ldots, x^{n-1}\right) \in \mathbb{R}^{n} \mid x^{0}>0\right\} .
$$

\begin{tabular}{|c|c|c|c|c|}
\hline$V$ & & Type I & Type II & Type III $(m=2 \ell)$ \\
\hline \multirow[t]{3}{*}{$\operatorname{Sym}(m, \mathbb{R})$} & $G$ & $S L(m, \mathbb{R}) \times \mathbb{R}_{+}$ & $x$ & $S p(\ell, \mathbb{C})$ \\
\hline & $H$ & $S O(p, q)$ & $\times$ & $S p(\ell, \mathbb{R})$ \\
\hline & $K$ & $S O(m)$ & $x$ & $S p(\ell)$ \\
\hline \multirow[t]{3}{*}{$\operatorname{Herm}(m, \mathbb{C})$} & $G$ & $S L(m, \mathbb{C}) \times \mathbb{R}_{+}$ & $S O(m, m)$ & $S p(\ell, \ell)$ \\
\hline & $H$ & $S U(p, q)$ & $S O(m, \mathbb{C})$ & $S p(\ell, \mathbb{C})$ \\
\hline & $K$ & $S U(m)$ & $S O(m) \times S O(m)$ & $S p(\ell) \times S p(\ell)$ \\
\hline \multirow[t]{3}{*}{$\operatorname{Herm}(m, \mathbb{H})$} & $G$ & $S L(m, \mathbb{H}) \times \mathbb{R}_{+}$ & $S O(2 m, \mathbb{C})$ & $\times$ \\
\hline & $H$ & $S p(p, q)$ & $S O^{*}(2 m)$ & $\times$ \\
\hline & $K$ & $S p(m)$ & $S O(2 m)$ & $x$ \\
\hline \multirow[t]{3}{*}{$\operatorname{Herm}(3, \mathbb{O})$} & $G$ & $E_{6(-26)} \times \mathbb{R}_{+}$ & $S U^{*}(8)$ & $x$ \\
\hline & $H$ & $F_{4}, F_{4(-20)}$ & $S p(3,1)$ & $\times$ \\
\hline & $K$ & $F_{4}$ & $S p(4)$ & $\times$ \\
\hline \multirow[t]{3}{*}{$\mathbb{R}^{1, n-1}$} & $G$ & $S O(1, n-1) \times \mathbb{R}_{+}$ & $S O(1, p) \times S O(1, q)$ & $S O(1, n)$ \\
\hline & $H$ & $S O(n-1), S O(1, n-2)$ & $S O(1, q-1) \times S O(1, q-1)$ & $S O(1, n-1)$ \\
\hline & $K$ & $S O(n-1)$ & $\begin{array}{c}S O(p) \times S O(q) \\
(1<p<n)\end{array}$ & $S O(n)$ \\
\hline
\end{tabular}

Il est isomorphe à l'hyperboloïde d'équation

$$
-\left(y^{1}\right)^{2}-\cdots-\left(y^{n-1}\right)^{2}+\left(y^{n}\right)^{2}-\left(y^{n+1}\right)^{2}=1,
$$

l'isomorphisme étant défini par

$$
y^{1}=\frac{x^{1}}{x^{0}}, \ldots, y^{n-1}=\frac{x^{n-1}}{x^{0}}, y^{n}=\frac{1+\|x\|^{2}}{2}, y^{n+1}=\frac{1-\|x\|^{2}}{2} .
$$




\begin{tabular}{ccc}
\hline$V_{0}$ & & Type IV \\
\hline $\operatorname{Sym}(m, \mathbb{R})$ & $G$ & $S p(m, \mathbb{R})$ \\
& $H$ & $S L(m, \mathbb{R}) \times \mathbb{R}_{+}$ \\
& $K$ & $U(m)$ \\
\hline $\operatorname{Herm}(m, \mathbb{C})$ & $G$ & $S U(n, n)$ \\
& $H$ & $S L(m, \mathbb{C}) \times \mathbb{R}_{+}$ \\
& $K$ & $S(U(m) \times U(m))$ \\
\hline $\operatorname{Herm}(m, \mathbb{H})$ & $G$ & $S O^{*}(4 m)$ \\
& $H$ & $S L(m, \mathbb{H}) \times \mathbb{R}_{+}$ \\
& $K$ & $U(2 m)$ \\
\hline$H_{e r m}(m, \mathbb{O})$ & $G$ & $E_{7}(-25)$ \\
& $H$ & $E_{6}(-26) \times \mathbb{R}+$ \\
& $K$ & $E_{6} \times \mathbb{T}$ \\
\hline $\mathbb{R}^{1, n-1}$ & $G$ & $S O(2, n)$ \\
& $H$ & $S O(1, n-1) \times \mathbb{R}_{+}$ \\
& $K$ & $S O(n) \times \mathbb{T}$ \\
\hline & &
\end{tabular}

\section{Bibliographie}

S. Ben Saïd (2000), Weighted Bergman spaces on bounded symmetric domains, preprint.

W. Bertram (1996a), Un théorème de Liouville pour les algèbres de Jordan, Bull. Soc. Math. France 124, 299-327.

W. Bertram (1996b), On some causal conformal groups, J. Lie Theory 6, 215-247.

W. Bertram (1998), Algebraic structures of Makarevic spaces. I, Transformation Groups 3, 3-32.

G. van Dijk and M. Pevzner (2001), Berezin kernels on tube domains, J. Funct. Anal. 181, $189-208$.

M. Engliš (1999), A mean value theorem on bounded symmetric domains, Proc. Amer. Math. Soc. 127, 3259-3268.

J. Faraut and A. Korányi (1994), Analysis on Symmetric Cones, Oxford University Press.

S. Gindikin and S. Kaneyuki (1998), On the automorphism group of the generalized conformal structure of a symmetric R-space, Differential Geom. Appl. 8, 21-33.

J. Hilgert and G. Ólafsson (1997), Causal Symmetric Spaces, Academic Press.

I. L. Kantor (1967), Nonlinear transformation groups defined by general norms of Jordan algebras, Soviet Math. Dokl. 8, 176-180.

J.-B. Kayoya (1994), Analyse sur les algèbres de Jordan simples réelles, thèse.

Y. Khlif (2000), Intégrales de Riesz sur un espace symétrique de type Cayley, thèse.

A. Unterberger and H. Upmeier (1994), The Berezin transform and invariant differential operators, Comm. Math. Phys. 164, 563-597. 